\title{
Predictive Value of Hypoxemia in the Diagnosis of Pneumonia in the Pediatric Population
}

\author{
Saleh Al Harbi
}

\author{
Department of Pediatrics, Umm Al-Qura University, Mecca, Saudi Arabia, and Department of Pediatric, \\ Dr. Soliman Fakeeh Hospital, Jeddah - 23323, Saudi Arabia; sah508@hotmail.com
}

\begin{abstract}
Pneumonia is the leading cause of morbidity and mortality in children less than 5 years of age ${ }^{1}$. According to a systematic review, hypoxaemia as detected with pulse oximetry has been observed in $13 \%$ of children with WHO-defined pneumonia requiring hospitalisation (severe and very severe classifications). This corresponds to at least 1.5 to 2.7 million annual cases of hypoxaemic pneumonia presenting to health-care facilities ${ }^{2}$. This study was aimed to identify the clinical predictive values of hypoxemia in children with radiographic evidence of Pneumonia. A retrospective cohort study was conducted at Dr. Soliman Fakeeh Hospital. The aim of the study was to seek an association between pulse oxygen saturation and radiographic pneumonia in the pediatric age group. Data was collected and analyzed in IBM SPSS Version 20. The results were significant for hypoxemia across all age groups, $\left[\mathrm{x}^{2}(1, \mathrm{~N}=755)=10.327, \mathrm{p}=.001\right]$. Approximately $94 \%$ of patients with hypoxemia had pneumonia. Due to its statistical significance, evaluation of oxygen saturation for hypoxemia may be useful in the assessment of pneumonia risk among children in settings with underprivileged resources.
\end{abstract}

Keywords: Hypoxemia, Pediatrics, Pneumonia, Predictive Value

\section{Introduction}

Pneumonia is the leading cause of morbidity and mortality in children less than 5 years of age ${ }^{1}$. Despite the high incidence of childhood pneumonia worldwide, data is limited regarding the ability to diagnose and predict the clinical course of pneumonia ${ }^{4}$. Studies on the values hypoxemia thresholds in the diagnosis of pneumonia in children are limited ${ }^{5}$. As of 2015, it has been estimated that approximately 120-156 million cases of acute lower respiratory tract infections occur worldwide and result in 1.4 million deaths with more than $95 \%$ of the morbidities in lower and middle income countries ${ }^{6,7}$. In a recent study, it was found that children under the age of five years suffering from severe pneumonia associated with hypoxia had a higher chance of having age related tachypnea ${ }^{8}$. The clinical outcome of hypoxemic children is greatly improved with early recognition and treatment ${ }^{6}$.

We therefore found it appropriate to conduct this study to identify the clinical predictive values of hypoxemia in children with radiographic evidence of pneumonia.

\section{Methods}

\subsection{Study Design}

A retrospective cohort study was conducted at Dr. Soliman Fakeeh Hospital in the port city of Jeddah, Saudi Arabia. The aim of the study was to seek an association between pulse oxygen saturation and radiographic pneumonia in the pediatric age group.

\subsection{Participants and Enrollment}

Data was extracted retrospectively from, OASIS, the hospital electronic medical record operating system, between the dates of January 1, 2014 to December 18, 2018. Inclusion criteria were any pediatric patient that presented to the hospital with documented respiratory rate and pulse oxygen saturation and with physician diagnosed Pneumonia. Patients were excluded from the study if a chest $\mathrm{x}$-ray was not done to show evidence of Pneumonia.

${ }^{*}$ Author for correspondence 


\subsection{Outcomes and Definitions}

Patients were stratified into five categories based on age related respiratory rates as determined by the 2018 PALS study guide for age related respiratory rates $(\mathrm{RR})^{9}$. The age categories were: less than one year of age (infant), one to three years (toddler), four to five years (preschooler), six to 12 years (school aged child), and 13 to 18 years of age (adolescent). Hypoxemia was considered as pulse oxygen saturation less than $95 \%$ across all age groups.

\subsection{Data Collection and Analysis}

Data was collected and analyzed in IBM SPSS Statistics version 20. Pearson Chi square and Fisher's exact appropriate tests were applied to compare the rate of radiological pneumonia among children with and without hypoxemia. Results were defined in terms of sensitivity, specificity, Positive Predictive Value (PPV) and Negative Predictive Value (NPV). A probability below 0.05 was regarded as statistically significant. The strength of association of hypoxemia with radiological evidence of Pneumonia was determined by the Phi correlation coefficient. Each age group was analyzed for association of SPO2 with age related RR using the Chi-square test.

\section{Results}

A total of 755 patients were enrolled in the study and were diagnosed to have clinical Pneumonia based on history and physical examination. The toddler age group constituted approximately $44 \%$ of the studied population and infants were around $29 \%$. The adolescent age group did not contribute significantly to the study since only one participant qualified (Figure 1).

The study showed that $85 \%$ of those enrolled were found to have radiographic evidence of Pneumonia. Of the 755 patients enrolled, only 135 were found to have hypoxemia (18\%).

All hypoxemic infants had pneumonia and tachypnea. 95.7\% of infants with hypoxemia had pneumonia despite normal RR. There was a statistical significance in infant oxygen saturation and radiographic evidence of pneumonia despite a normal $\mathrm{RR}\left[\mathrm{G}^{2}(1, \mathrm{~N}=188)=3.879\right.$, $\mathrm{p}=.049]$. More than $90 \%$ of toddlers who were hypoxemic had pneumonia regardless of RR. In the school age category, all children enrolled in the study and were tachypneic had radiographic evidence of pneumonia

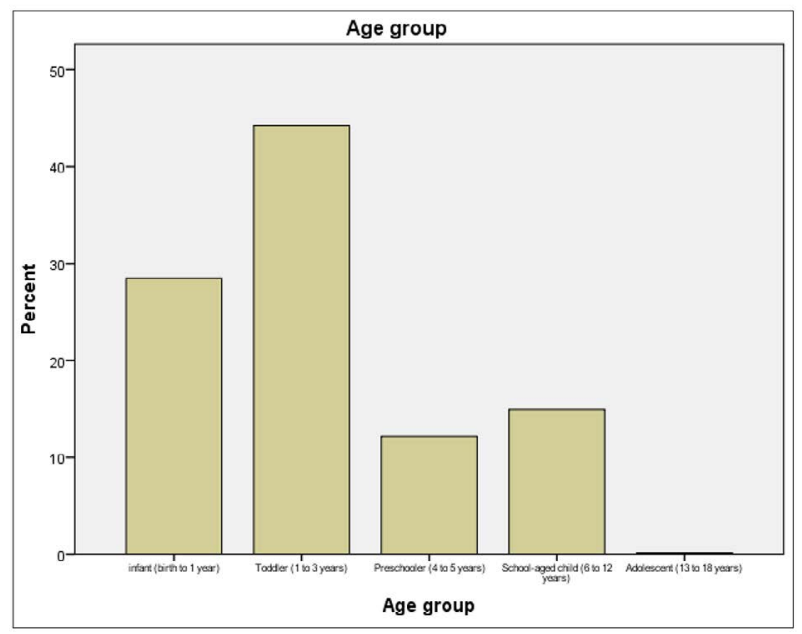

Figure 1. Distribution of participants based on age.

therefore no data exists for the specificity and negative predictive value in this subgroup. Overall, the results were significant for hypoxemia across all age groups, $\left[\mathrm{x}^{2}(1\right.$, $\mathrm{N}=755)=10.327, \mathrm{p}=.001$ ]. Approximately $94 \%$ of patients with hypoxemia had pneumonia. Although the $p$ value for hypoxemia was largely statistically significant in all age categories the phi correlation coefficient for hypoxemia was ' 117 '. This value cor relates with a slightly positive association of hypoxemia with radiographic evidence of Pneumonia (Table 1).

\section{Discussion}

We retrospectively examined the association between hypoxemia with radiographic pneumonia among 755 children presenting to our hospital who had a CXR obtained for possible pneumonia. Hypoxemia was positively associated with radiographic pneumonia across all age groups. The majority of hypoxemic toddlers had pneumonia regardless of RR.

The systematic review and meta-analysis conducted ${ }^{6}$ supported the regular use of pulse oxygen saturation to aid in recognition children with increased morbidity due to acute lower respiratory tract infections.

The generalizability of our study may be limited because our study was conducted in a region of Saudi Arabia where there is widespread access to healthcare and clinicians are more likely to see patients earlier in the course of their illness and with less advanced pneumonias. In addition, the port city of Jeddah accommodates a massive variety of expatriates not found elsewhere in the 
Table 1. Distribution of signs based on sensitivity and specificity

\begin{tabular}{|c|c|c|c|c|c|}
\hline Sign & Sensitivity \% & Specificity \% & PPV \% & NPV \% & P-Value \\
\hline SPO2 & 19.8 & 92.9 & 94.1 & 16.8 & .001 \\
\hline \multicolumn{6}{|l|}{ Age Group: Infant } \\
\hline SPO2 and Tachypnea $(\mathrm{N}=27)$ & 57.7 & 100 & 100 & 8.3 & .196 \\
\hline SPO2 and Normal RR $(\mathrm{N}=188)$ & 14.1 & 96.9 & 95.7 & 18.8 & .049 \\
\hline \multicolumn{6}{|l|}{ Age Group: Toddler } \\
\hline SPO2 and Tachypnea $(\mathrm{N}=81)$ & 44.7 & 40 & 91.9 & 4.5 & .507 \\
\hline SPO2 and Normal RR $(\mathrm{N}=253)$ & 11.1 & 97.8 & 95.8 & 19.2 & .035 \\
\hline \multicolumn{6}{|l|}{ Age Group: Preschooler } \\
\hline SPO2 and Tachypnea $(\mathrm{N}=19)$ & 35.3 & 50 & 85.7 & 8.3 & .688 \\
\hline SPO2 and normal RR $(\mathrm{N}=73)$ & 6.7 & 92.3 & 80 & 17.6 & .896 \\
\hline \multicolumn{6}{|l|}{ Age Group: School Age Child } \\
\hline SPO2 and Tachypnea $(\mathrm{N}=15)$ & 80 & - & 100 & - & - \\
\hline SPO2 and Normal RR $(\mathrm{N}=98)$ & 13.1 & 92.9 & 91.7 & 15.1 & .295 \\
\hline
\end{tabular}

country and it is considered as the main entryway to the pilgrims hosted by Saudi Arabia, therefore the sample size in our study may not be reflective of the population in other areas of the country.

Moreover, we did not exclude children with underlying medical conditions that may affect the respiratory rate such as congenital heart disease and neuromuscular disorders. Although some patients presented with symptoms indicative of pneumonia such as fever or cough, patients that did not undergo chest radiography were not included in the study. Furthermore, respiratory rate was not adjusted for temperature.

Since there is no ideal standard for the definition of pneumonia we included equivocal radiographs to decrease the chance of missing a child with radiographic pneumonia. Due to the minimal enrollment in the adolescent age group, we did not have the statistical power to evaluate the association between tachypnea and radiographic pneumonia among children between 13 to 18 years of age.

\section{Conclusion}

In the modern health care setting, hypoxemia is not a sensitive indicator of pneumonia. However, due to its statistical significance, evaluation of oxygen saturation for hypoxemia may be useful in the assessment of pneumonia risk among children in settings with underprivileged resources. Further studies should focus on more rural areas of Saudi Arabia with fewer medical resources and should include a larger sample. Medical facilities should strive to have pulse oximetry available in all areas.

\section{Acknowledgments}

Funding: no funding sources

Conflict of Interest: none

Ethical Approval: Yes

\section{References}

1. Rudan, et al. Epidemiology and Etiology of Childhood Pneumonia. Bulletin of the World Health Organization, World Health Organization. 1 May 2008. www.scielosp.org/ article/bwho/2008.v86n5/408-416B/. https://doi.org/10.24 71/BLT.07.048769. PMid: 18545744, PMCid: PMC2647437.

2. Subhi R, Adamson M, Campbell H, Weber M, Smith K, Duke T. Hypoxaemia in Developing Countries Study Group. The prevalence of hypoxaemia among ill children in developing countries: A systematic review. Lancet Infect Dis. 2009; 9:219-27. https://doi.org/10.1016/S14733099(09)70071-4.

3. Florin, T. A., et al. Variation in Emergency Department Diagnostic Testing and Disposition Outcomes in Pneumonia. Pediatrics. 2013; 132(2):237-244. DOI:10.1542/ peds.2013-0179. https://doi.org/10.1542/peds.2013-0179. PMid: 23878049.

4. Shah, Sonal, et al.Lack of Predictive Value of Tachypnea in the Diagnosis of PneumoniainChildren. The PediatricInfectious Disease Journal. 2010; 29(5):406-409. doi:10.1097/in 
f.0b013e3181cb45a7.https://doi.org/10.1097/INF.0b013e31 81cb45a7. PMid: 20032805.

5. Kushwah, Meenakshi S., et al. Clinical Predictors of Hypoxemia in Children with WHO Classified Pneumonia. International Journal of Contemporary Pediatrics. 2018; 5(4):1176. doi:10.18203/2349-3291.ijcp20181981. https:// doi.org/10.18203/23493291.ijcp20181981.

6. Lazzerini, Marzia, et al. Hypoxaemia as a Mortality Risk Factor in Acute Lower Respiratory Infections in Children in Low and Middle-Income Countries: Systematic Review and Meta-Analysis. PloS One, Public Library of Science. 15 Sept. 2015. www.ncbi.nlm.nih.gov/pmc/articles/PMC 4570717/.

7. Alwadhi, Varun, et al. Tachypnea and Other Danger Signs vs Pulse Oximetry for Prediction of Hypoxia in Severe
Pneumonia/Very Severe Disease. Indian Pediatrics. 2017; 54(9):729-734. doi:10.1007/s13312-017-1163-6. https://doi. org/10.1007/s13312-017-1163-6. PMid: 28607210.

8. Rudan, et al. Epidemiology and Etiology of Childhood Pneumonia. Bulletin of the World Health Organization, World Health Organization. 1 May 2008. www.scielosp.org/ article/bwho/2008.v86n5/408-416B/.

9. Aehlert, Barbara. PALS: Pediatric Advanced Life Support Study Guide. Jones \& Bartlett Learning, 2018, p.7.

10. Weber M, Usen S, Palmer A et al. Predictors of hypoxemia in hospital admission with acute respiratory tract infection in a developing country. Arch Dis Child. 1997; 76:310-14. 16. Oxygen therapy. Management of the child with. PMid: 9166021, PMCid: PMC1717166. https://doi.org/10.1136/ adc.76.4.310. 